\title{
Short Circuit Fault Analysis of Electrical Power System using MATLAB
}

\author{
Daljeet Kaur", Dr. S.K.Bath ${ }^{* *}$, Darshan Singh Sidhu \\ *, ${ }^{* *}$ Department of Electrical Engineering, PTUGZSCampus, Bathinda. \\ ${ }^{* * *}$ Department of Electronics and Communication Engineering,PTUGZSCampus,Bathinda.
}

\begin{abstract}
The objective of the present study is to simulate short circuit faults on different buses of a power system network and to estimate the state of the power system before and after a fault, which includes various bus voltages and current flow on various transmission lines. The analysis before the fault is carried out by solving non-linear load flow problem by using numerical iterative technique of Newton-Raphson method. State estimation after short circuit fault is carried by using algorithm of short circuit current computation. The short circuit currents dictate the rating of circuit breakers to be employed at various buses and in various lines of the network. The effect of three phase short circuit faults on power system has been studied in terms of post-fault conditions and pre-fault conditions. In this paper, three phase short circuit fault is simulated on the standard IEEE 11 bus and IEEE 30 bus system and fault current level is calculated and short circuit MVA rating for the circuit breaker has been chosen. The analysis of power system under faulty conditions is important to determine the values of system voltages and currents during faulted conditions, so that protective devices may be set to minimize the harmful effects of such contingencies.
\end{abstract}

Keywords: Load flow, Short circuit current, SCMVA ratings of circuit breakers, Three phase fault calculation.

\section{INTRODUCTION}

Electrical energy consumption increases more and more on a daily basis. Technological development is the reason for the use of electrical energy. Every year many more power stations, transmission lines and substations are constructed. This situation increases the fault current levels in power systems. A power system is not static but changes during operation (switching on or off of generators and transmission lines) and during planning (addition of generators and transmission lines). Thus fault studies need to be routinely performed by utility engineers [1].

A Fault is defined as any failure which interferes with the normal current flow[2]. Various types of faults occur on power system are shown in fig. 1 Short circuit fault is a fault in which current flow bypasses the normal load and an Open circuit fault occurs if a circuit is interrupted by some failure. Fault analysis can be broadly grouped into symmetrical and unsymmetrical faults. A balanced three phase fault occurs when there is a simultaneous short circuit across all three phases. This type of fault is also called Symmetrical fault. If only some phases are affected, the resulting Unsymmetrical fault [1][3]. Majority of fault occurring on power system are unsymmetrical faults, however, the circuit breaker rated MVA breaking capacity is based on three-phase symmetrical faults. The reason is that a three-phase fault produces the greatest fault current and Circuit Breaker must be capable of interrupting it[4].

The causes of faults are numerous and they include lightning, insulation aging, heavy winds, trees falling across lines, vehicles colliding with poles, birds, kites, etc. The effects of faults on power system are:

(i) Overheating and mechanical forces developed by faults may damage the electrical equipment such as busbars, generators and transformers.

(ii) The voltage profile of the system may be reduced to unacceptable limits as a result of fault. A frequency drop may lead to instability [5].

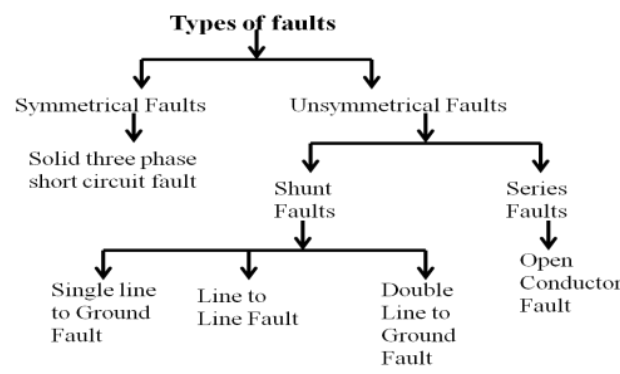

Figure 1: Various Types of Faults that occurs in Power System 


\subsection{USE OF SIMULATION SOFTWARE}

In this paper, short circuit fault analysis on power system is done by using MATLAB programming. MATLAB is a powerful software package used for high performance scientific numerical computation, data analysis and visualization.

The single line diagram for standard IEEE 11 bus and IEEE 30 bus system is shown in Fig. 2 and Fig.3.

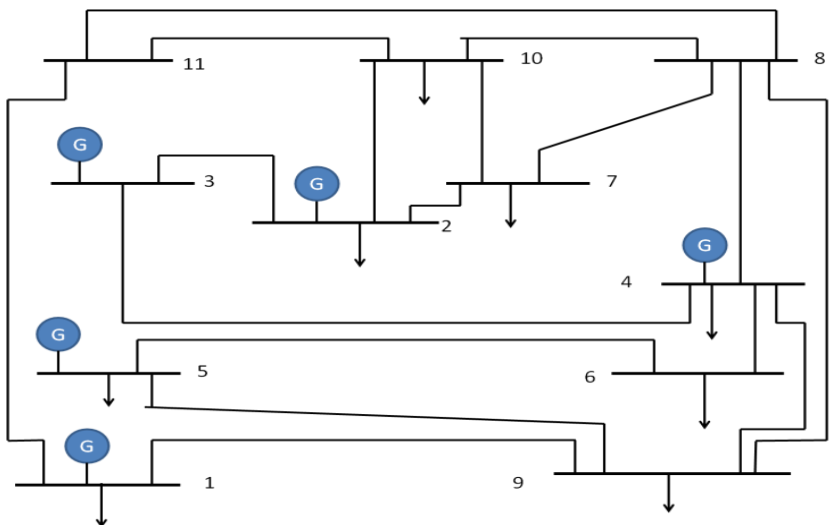

Figure2: One line diagram for 11 bus system

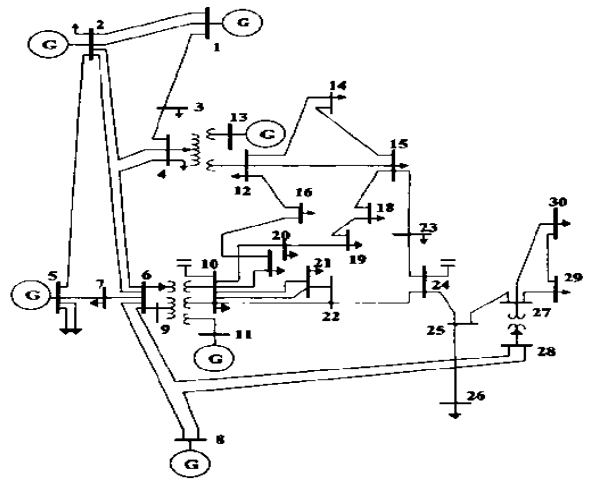

Figure3: One line diagram for IEEE 30 bus

\section{2 \\ NEED FOR FAULT ANALYSIS IN PLANNING AND OPERATION OF POWER SYSTEM}

The system being planned is to be optimal with respect to construction cost, performance and operating efficiency. For this better planning tools are required. In general, the major power system tools are: load Flow Analysis, short circuit analysis or fault calculations, stability analysis etc.

The purpose of an electrical power system is to generate and supply electrical energy to consumers with reliability and economy. The greatest threat to this purpose of a power system is the short circuit [3]. The evaluation of fault currents on a power system is significant because the protective devices to be installed on the system depend on the values of the fault currents. Information gained from fault analysis used to select the appropriate size and type of protective equipments to be installed on the system so that the continuity of supply is ensured even when there is a fault on the power system.

\subsection{PRELIMINARY CALCULATIONS}

\section{Short Circuit Fault Analysis Problem Formulation}

In the fault studies, it is necessary to have the knowledge of pre-fault voltages and currents. These prefault conditions can be obtained from the results of load flow studies by the Newton Raphson method. Load flow analysis is a technique that provides basic calculation procedure in order to determine the characteristics of power system under steady state condition [8]. A solution of the power flow problem using Newton Raphson method is depicted in Fig. 4. The Newton Raphson method is adopted for large networks due to its quadratic convergence characteristics, high accuracies obtained in a few iterations and no. of iterations independent of the size of the system [4].

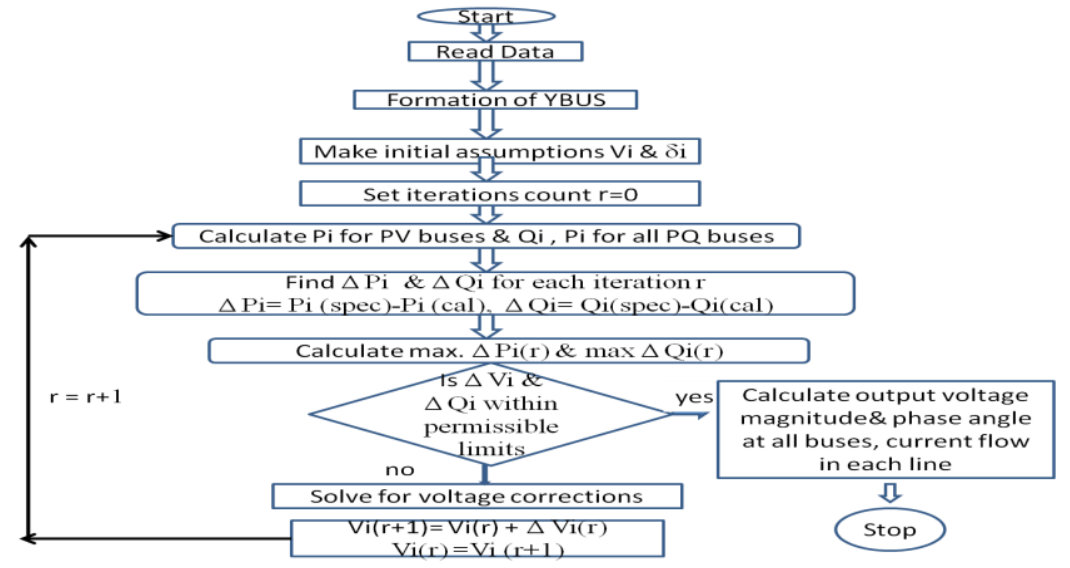

Figure 4: Flow Chart for Newton Raphson Load Flow Method 


\subsection{CALCULATION OF SHORT CIRCUIT CURRENTS}

If the insulation of system fails at any point or if two or more conductor that normally operates with a potential difference comes in contact with each other, a short circuit is said to be occur. Fault analysis calculate the fault currents magnitudes that are used to determine short circuit megavolt ampere(SCMVA) ratings of appropriate circuit breakers[9][10]. For a symmetrical fault, the negative and zero sequences are absent. The positive sequence present and modified for fault analysis as shown in Fig.5:

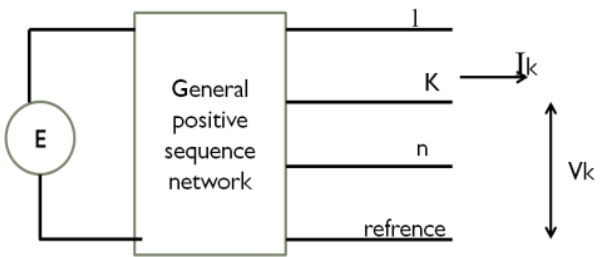

Figure 5: Positive Sequence Network Modified for Fault Analysis[11]

The injected bus currents in terms of bus voltages for a n-bus network is calculated as;

$\mathrm{I}_{\text {bus }}=\mathrm{Y}_{\text {bus }} \cdot \mathrm{V}_{\text {bus }}$

Where $\mathrm{I}_{\text {bus }}$ is the bus current vector entering the bus \& $\mathrm{Y}_{\text {bus }}$ is the bus admittance matrix

For a fault at bus $\mathrm{k}$, the current entering every bus except the faulted bus $\mathrm{k}$ is zero thus equation (1) becomes:

$\left[\begin{array}{c}0 \\ 0 \\ \vdots \\ -\mathrm{Ik}(\mathrm{F}) \\ 0\end{array}\right]=\left[\begin{array}{cccc}Y 11 & \ldots & Y 1 k & Y 1 n \\ Y 21 & \ldots & Y 2 k & Y 2 n \\ \vdots & \ddots & \vdots & \vdots \\ Y k 1 & \ldots & Y k k & Y k n \\ Y n 1 & \ldots & Y n k & Y n n\end{array}\right]\left[\begin{array}{c}\Delta V 1 \\ \Delta V 2 \\ \vdots \\ \Delta V k \\ \Delta V n\end{array}\right]$

Equation (2) can be written as

$\mathrm{I}_{\text {bus }}(\mathrm{F})=\mathrm{Y}_{\text {bus }} \cdot \Delta \mathrm{V}_{\text {bus }}$

From above equation ;

$\Delta \mathrm{V}_{\text {bus }}=\operatorname{inverse}\left(\mathrm{Y}_{\text {bus }}\right) . \mathrm{I}_{\text {bus }}(\mathrm{F})$

$$
=\mathrm{Z}_{\text {bus }} \cdot \mathrm{I}_{\mathrm{bus}}(\mathrm{F})
$$

$\mathrm{Z}_{\text {bus }}=$ inverse $\left(\mathrm{Y}_{\text {bus }}\right)$ is the bus impedance matrix

$\mathrm{V}_{\text {bus }}(\mathrm{F})=\mathrm{V}_{\text {bus }}(0)+\Delta \mathrm{V}_{\text {bus }}$

Substituting (4) into equation (5) gives;

$\mathrm{V}(\mathrm{F})=\mathrm{V}_{\text {bus }}(0)+\mathrm{Z}_{\text {bus }} . \mathrm{I}_{\text {bus }}(\mathrm{F})$

Above equation (6) can be written in matrix form as;

$$
\left[\begin{array}{c}
V 1(F) \\
V 2(F) \\
\vdots \\
V k(F) \\
V n(F)
\end{array}\right]=\left[\begin{array}{c}
V 1(0) \\
V 2(0) \\
\vdots \\
V k(0) \\
V n(0)
\end{array}\right]+\left[\begin{array}{cccc}
Z 11 & \ldots & Z 1 k & Z 1 n \\
Z 21 & \ldots & Z 2 k & Z 2 n \\
\vdots & \ddots & \vdots & \vdots \\
Z k 1 & \ldots & Z k k & Z k n \\
Z n 1 & \ldots & Z n k & Z n n
\end{array}\right]\left[\begin{array}{c}
0 \\
0 \\
\vdots \\
-\mathrm{Ik}(\mathrm{F}) \\
0
\end{array}\right]
$$

The voltage at bus $\mathrm{k}$ during the fault is $\mathrm{Vk}(\mathrm{F})$. So;

$\mathrm{V}_{\mathrm{k}}(\mathrm{f})=\mathrm{V}_{\mathrm{k}}(0)-\mathrm{Z}_{\mathrm{kk}} \cdot \mathrm{I}_{\mathrm{k}}(\mathrm{F})$

But $V_{k}(F)=Z^{f} . I_{k}(F)$, where $Z^{f}$ is fault impedance \& Where $Z_{k k}$ is $k^{\text {th }} k^{\text {th }}$ element of bus impedance matrix and this equation put in above equation (7)

Equation (7) becomes;

$\mathrm{Z}^{\mathrm{f}} . \mathrm{I}_{\mathrm{k}}(\mathrm{F})=\mathrm{V}_{\mathrm{k}}(0)-\mathrm{Z}_{\mathrm{kk}} \cdot \mathrm{I}_{\mathrm{k}}(\mathrm{F})$

Solving for $\mathrm{I}_{\mathrm{k}}(\mathrm{F})$ gives;

$\mathbf{I}_{\mathbf{k}}(\mathbf{F})=\frac{\mathbf{V k}(\mathbf{0})}{(\mathbf{Z k k}+\mathbf{Z f})}$

For bolted or solid fault, $Z^{\mathrm{f}}=0 \quad[12]$

For any bus $i$ the bus voltage during fault is;

$\mathrm{V}_{\mathrm{i}}(\mathrm{F})=\mathrm{V}_{\mathrm{i}}(0)-\mathrm{Z}_{\mathrm{ik}} \cdot \mathrm{I}_{\mathrm{k}}(\mathrm{F})$, where $\mathrm{Z}_{\mathrm{ik}}$ is i $\mathrm{i}^{\text {th }} \mathrm{k}^{\text {th }}$ element of bus impedance matrix.

From equation $(8)$;

$\mathbf{V}_{\mathbf{i}}(\mathbf{F})=\mathbf{V}_{\mathbf{i}}(\mathbf{0})-\mathbf{Z}_{\mathbf{i k}} \cdot \frac{\mathbf{V k}(\mathbf{0})}{(\mathbf{Z k k}+\mathbf{Z f})}$

The short circuit current in the line connected between bus $i$ and $j$ is;

$\mathbf{I}_{\mathbf{i j}}(\mathbf{F})=\frac{\mathbf{V i}(\mathbf{F})-\mathbf{V j}(\mathbf{F})}{\mathbf{z i j}}$ where zij is series impedance when line connected between buses $\mathbf{i}$ and $\mathbf{j}$.

Flow Chart for the Calculations of Three-Phase Short Circuit Fault is shown in figure 6. 


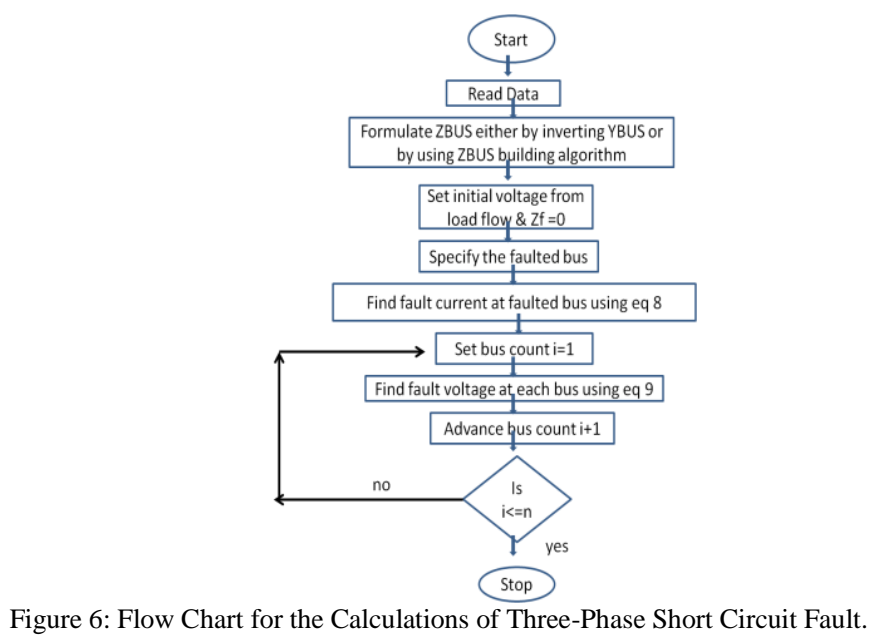

\subsection{SELECTION OF CIRCUIT BREAKERS}

The circuit breaker has to perform the following major duties under short circuit conditions;

1) To open the contacts to clear the fault.

2) To close the contacts onto a fault.

3) To carry fault current for a short time while another circuit breaker is clearing the fault.

In addition to the rated voltage, current and frequency, circuit breakers have the following important ratings:

1) Breaking capacity 2) Making capacity 3) Short time capacity

The Breaking capacity of a circuit breaker is of two types i.e. Symmetrical Breaking capacity and Asymmetrical Breaking capacity.

Symmetrical breaking capacity: It is the r.m.s value of the ac component of the fault current that the circuit breaker is capable of breaking under specified conditions of recovery voltage.

Asymmetrical breaking capacity: It is the r.m.s value of the total current comprising of both ac and dc components of the fault current that the $\mathrm{CB}$ can break under specified conditions of recovery voltage.

The breaking capacity of a CB is generally expressed in MVA.

If voltage \& current in p.u. values on a 3 ph. Basis, then

$\operatorname{SCMVA}(3$ ph $)=\mid$ Vprefault $|*|$ Isc $\mid *($ MVA base $)$

Rated MVA interrupting capacity of a CB is to be more than or equal to the short circuit MVA required to be interrupted[4].

The rated asymmetrical breaking capacity is 1.6 times the rated symmetrical current.

Making capacity: The rated making current is defined as the peak value of the current including dc component in the first cycle at which a CB can be closed onto a short circuit.

Making current $=\sqrt{ } \mathbf{2} * 1.8 *$ symmetrical breaking current

The multiplication by $\sqrt{2}$ is obtain the peak value and again by 1.8 to take the d.c. component into account. Short-time current rating : It is the r.m.s value of total current that the CB can carry safely for a specified short period.

Short time current rating=Breaking current / rated normal current[13].

\subsection{A COMPUTATIONAL ALGORITHM FOR SHORT CIRCUIT STUDIES}

Algorithm adopted for this type of analysis consists following steps;

STEP 1: Obtain pre-fault voltages at all buses and currents in all lines through a load flow study.

STEP 2: Find Bus impedance matrix by inverting the bus admittance matrix.

STEP 3: Choose MVAbase, KVbase \& calculate Ibase.

STEP 4: Specify the faulty bus and obtain current at the faulty bus using equation (8) and bus voltages during fault at all buses using equation (9).

STEP 5: Find current flows in each line of the system using equation (10).

STEP 6: Calculate SCMVA rating of circuit breaker(choose acc. to the fault current magnitude ) for each line \& at each bus using equation (11). 


\section{Results And Discussion \\ 3.1 RESULTS FOR IEEE 11 BUS 17 TRANSMISSION LINE SYSTEM}

Firstly, discuss the results for load flow for IEEE 11 bus system. The load flow analysis was carried out using the Newton-Raphson load flow method. This analysis determines the voltage magnitude, phase angle in degree, real power and reactive powers at each bus and current flows in the transmission lines. The result of the load flow is shown in Table 1 and Table 2. It can be observed that the voltage magnitudes are within the tolerance ranges of $\pm 10 \%$.

Table 1: Voltage magnitude, phase Angle, Real and Reactive Powers from pre-fault analysis by Newton Raphson Method on Standard IEEE 11 bus system.

\begin{tabular}{|c|c|c|c|c|c|}
\hline Bus no. & $\begin{array}{l}\text { Voltage magnitude } \\
\text { (p.u) }\end{array}$ & $\begin{array}{l}\text { Voltage magnitude } \\
(\mathrm{KV})\end{array}$ & Angle (degree) & $\begin{array}{l}\text { Real Power } \\
\text { (p.u) }\end{array}$ & $\begin{array}{l}\text { Reactive Power } \\
\text { (p.u) }\end{array}$ \\
\hline 1 & 1.0700 & 70.6200 & 0.0000 & 0.4339 & 0.2639 \\
\hline 2 & 1.0924 & 72.1009 & 1.6622 & 0.6625 & 0.4989 \\
\hline 3 & 1.0950 & 72.2700 & 8.5206 & 0.6625 & -0.0504 \\
\hline 4 & 1.0620 & 70.0920 & 3.9906 & 0.4778 & 0.1539 \\
\hline 5 & 1.0460 & 69.0360 & 2.2811 & 0.4778 & 0.1461 \\
\hline 6 & 1.0501 & 69.3068 & 2.4221 & -0.1000 & -0.0200 \\
\hline 7 & 1.0118 & 66.7783 & -3.0409 & -0.4000 & -0.1000 \\
\hline 8 & 0.9890 & 65.2723 & -3.3570 & -0.9000 & -0.4500 \\
\hline 9 & 0.9983 & 65.8876 & -1.5303 & -0.7000 & -0.3500 \\
\hline 10 & 1.0326 & 68.1517 & -1.9984 & -0.2500 & -0.0500 \\
\hline 11 & 1.0310 & 68.0450 & -2.4009 & -0.2500 & -0.0500 \\
\hline
\end{tabular}

Table 2: Line current magnitudes from load flow analysis using Newton Raphson Method on IEEE 11 bus

\begin{tabular}{llll}
\hline Line no. & \multicolumn{1}{c}{ system. } & \\
\hline 1 & From bus - To bus & CurrentMagnitude (p.u) & Current Magnitude (A) \\
2 & $1-9$ & 0.0129 & 11.2668 \\
3 & $1-11$ & 0.0099 & 8.6243 \\
4 & $2-3$ & 0.0219 & 19.1902 \\
5 & $2-7$ & 0.0198 & 17.3179 \\
6 & $2-10$ & 0.0152 & 13.2654 \\
7 & $3-4$ & 0.0153 & 13.4033 \\
8 & $4-6$ & 0.0052 & 4.5841 \\
9 & $4-8$ & 0.0252 & 22.0357 \\
10 & $4-9$ & 0.0198 & 17.2849 \\
11 & $5-6$ & 0.0008 & 0.7106 \\
12 & $5-9$ & 0.0139 & 12.1762 \\
13 & $7-8$ & 0.0039 & 3.4424 \\
14 & $7-10$ & 0.0047 & 4.0925 \\
15 & $8-9$ & 0.0055 & 4.8421 \\
16 & $8-10$ & 0.0083 & 7.2988 \\
17 & $8-11$ & 0.0076 & 6.6374 \\
\hline
\end{tabular}

After the pre-fault calculations, a three phase short circuit fault was simulated on the 11 bus system then calculated the total fault current at each bus, fault voltage magnitude at each bus, fault voltage phase angle at each bus, fault current flows in the lines, SCMVA ratings based on the fault currents on each bus and lines was also calculated then the corresponding Circuit Breakers ratings are choosed. Total fault current at each bus and SCMVA ratings for that fault currents on each bus is shown in Table 3. The range of the circuit breakers at each bus determined for the 11 bus system is within 35MVA and 45MVA.

Table 3: Fault current magnitude, Short circuit MVA and Circuit breaker ratings at each bus.

\begin{tabular}{lllll}
\hline $\begin{array}{l}\text { Fault at bus } \\
\text { no. }\end{array}$ & $\begin{array}{l}\text { Fault } \\
\text { magnitude(p.u) }\end{array}$ & $\begin{array}{l}\text { Fault current } \\
\text { magnitude(A) }\end{array}$ & $\begin{array}{l}\text { SCMVA } \\
\text { (MVA) }\end{array}$ & $\begin{array}{l}\text { Circuit Rating (MVA) } \\
\text { Rreaker }\end{array}$ \\
\hline 1 & 0.3741 & 1963.5 & 40.0202 & 45 \\
2 & 0.3743 & 1964.5 & 40.8816 & 45 \\
3 & 0.3839 & 2014.9 & 42.0314 & 45 \\
4 & 0.3601 & 1890.0 & 38.2426 & 40 \\
5 & 0.3651 & 1916.2 & 38.1894 & 40 \\
6 & 0.3702 & 1943.1 & 38.8747 & 40 \\
7 & 0.3448 & 1809.7 & 34.8868 & 35 \\
8 & 0.3316 & 1740.4 & 32.7952 & 35 \\
9 & 0.3395 & 1781.9 & 33.8922 & 35 \\
10 & 0.3500 & 1837.1 & 36.1410 & 40 \\
11 & 0.3535 & 1855.3 & 36.4458 & 40 \\
\hline
\end{tabular}

Compare the pre-fault voltage magnitude in p.u. and fault voltage magnitude at each bus and it is observed that when a short circuit occurs, the voltage at faulted bus is reduced to zero and voltage magnitude on other buses 
are also effected, shown in Table 4 and also compare the pre-fault voltage angle in degree with the post-fault angle at each bus. It is seen that voltage angle at each bus increases when fault occur at different buses as shown in Table5.

Table 4: Effect on voltage magnitude in p.u after short circuit occurs at different buses.

\begin{tabular}{lllllllllllll}
\hline $\begin{array}{l}\text { Bus } \\
\text { no. }\end{array}$ & $\begin{array}{l}\text { Pre-fault } \\
\text { Voltage } \\
\text { (p.u) }\end{array}$ & $\begin{array}{l}\text { When } \\
\text { fault at } \\
\text { bus no. } \\
1\end{array}$ & $\begin{array}{l}\text { When } \\
\text { fault at } \\
\text { bus no. }\end{array}$ & $\begin{array}{l}\text { When } \\
\text { fault at } \\
\text { bus no. }\end{array}$ & $\begin{array}{l}\text { When } \\
\text { fault at } \\
\text { bus no. }\end{array}$ & $\begin{array}{l}\text { When } \\
\text { fault at } \\
\text { bus no. }\end{array}$ & $\begin{array}{l}\text { When } \\
\text { fault at } \\
\text { bus no. }\end{array}$ & $\begin{array}{l}\text { When } \\
\text { fault at } \\
\text { bus no. }\end{array}$ & $\begin{array}{l}\text { When } \\
\text { fault at } \\
\text { bus no. }\end{array}$ & $\begin{array}{l}\text { When } \\
\text { fault at } \\
\text { bus no. }\end{array}$ & $\begin{array}{l}\text { When } \\
\text { fault at } \\
\text { bus } \\
\text { no.10 }\end{array}$ & $\begin{array}{l}\text { When } \\
\text { fault at } \\
\text { bus } \\
\text { no.11 }\end{array}$ \\
\hline 1 & $\mathbf{1 . 0 7 0 0}$ & 0 & 0.0755 & 0.1134 & 0.0371 & 0.0486 & 0.0730 & 0.0236 & 0.0633 & 0.0422 & 0.0124 & 0.0255 \\
2 & $\mathbf{1 . 0 9 2 4}$ & 0.0558 & 0 & 0.0711 & 0.0156 & 0.0419 & 0.0569 & 0.0609 & 0.0884 & 0.0515 & 0.0468 & 0.0238 \\
3 & $\mathbf{1 . 0 9 5 0}$ & 0.0608 & 0.0386 & 0 & 0.0176 & 0.0322 & 0.0386 & 0.0462 & 0.0840 & 0.0578 & 0.0303 & 0.0215 \\
4 & $\mathbf{1 . 0 6 2 0}$ & 0.0886 & 0.0839 & 0.0894 & 0 & 0.0488 & 0.0515 & 0.0165 & 0.0531 & 0.0327 & 0.0162 & 0.0283 \\
5 & $\mathbf{1 . 0 4 6 0}$ & 0.0971 & 0.1090 & 0.1303 & 0.0463 & 0 & 0.0557 & 0.0214 & 0.0333 & 0.0377 & 0.0344 & 0.0411 \\
6 & $\mathbf{1 . 0 5 0 1}$ & 0.1013 & 0.1039 & 0.1164 & 0.0291 & 0.0359 & 0 & 0.0196 & 0.0364 & 0.0267 & 0.0315 & 0.0419 \\
7 & $\mathbf{1 . 0 1 1 8}$ & 0.1299 & 0.1091 & 0.1599 & 0.0902 & 0.1134 & 0.1304 & 0 & 0.0141 & 0.0319 & 0.0369 & 0.0614 \\
8 & $\mathbf{0 . 9 8 9 0}$ & 0.1489 & 0.1454 & 0.1836 & 0.1080 & 0.1295 & 0.1470 & 0.0495 & 0 & 0.0472 & 0.0680 & 0.0810 \\
9 & $\mathbf{0 . 9 9 8 3}$ & 0.1370 & 0.1532 & 0.1782 & 0.0961 & 0.0871 & 0.1215 & 0.0603 & 0.0143 & 0 & 0.0765 & 0.0815 \\
10 & $\mathbf{1 . 0 3 2 6}$ & 0.1062 & 0.0862 & 0.1397 & 0.0710 & 0.0942 & 0.1116 & 0.0071 & 0.0322 & 0.0167 & 0 & 0.0371 \\
11 & $\mathbf{1 . 0 3 1 0}$ & 0.0756 & 0.1078 & 0.1492 & 0.0744 & 0.0910 & 0.1120 & 0.0183 & 0.0287 & 0.0131 & 0.0274 & 0 \\
\hline
\end{tabular}

Table 5: Effect on voltage angle in degree after short circuit occurs at different buses.

\begin{tabular}{|c|c|c|c|c|c|c|c|c|c|c|c|c|}
\hline $\begin{array}{l}\text { Bus } \\
\text { no. }\end{array}$ & $\begin{array}{l}\text { Pre-fault } \\
\text { Voltage } \\
\text { (p.u) }\end{array}$ & $\begin{array}{l}\text { When } \\
\text { fault at } \\
\text { bus no. } \\
1\end{array}$ & $\begin{array}{l}\text { When } \\
\text { fault at } \\
\text { bus no. } \\
2\end{array}$ & $\begin{array}{l}\text { When } \\
\text { fault at } \\
\text { bus no. } \\
3\end{array}$ & $\begin{array}{l}\text { When } \\
\text { fault at } \\
\text { bus no. } \\
4\end{array}$ & $\begin{array}{l}\text { When } \\
\text { fault at } \\
\text { bus no. } \\
5\end{array}$ & $\begin{array}{l}\text { When } \\
\text { fault at } \\
\text { bus no. } \\
6\end{array}$ & $\begin{array}{l}\text { When } \\
\text { fault at } \\
\text { bus no. } \\
7\end{array}$ & $\begin{array}{l}\text { When } \\
\text { fault at } \\
\text { bus no. } \\
8\end{array}$ & $\begin{array}{l}\text { When } \\
\text { fault at } \\
\text { bus no. } \\
9\end{array}$ & $\begin{array}{l}\text { When } \\
\text { fault at } \\
\text { bus } \\
\text { no. } 10\end{array}$ & $\begin{array}{l}\text { When } \\
\text { fault at } \\
\text { bus } \\
\text { no. } 11\end{array}$ \\
\hline 1 & $\mathbf{0}$ & 0 & 166.64 & 164.97 & 156.93 & 151.86 & 154.35 & 34.24 & 5.715 & 13.736 & 58.96 & 11.02 \\
\hline 2 & 1.6622 & 153.47 & 0 & 161.85 & 121.56 & 139.59 & 144.71 & 6.7601 & 3.406 & 16.942 & 5.420 & 39.91 \\
\hline 3 & 8.5206 & 153.47 & 160.55 & 0 & 19.582 & 130.87 & 135.24 & 16.64 & 5.286 & 13.519 & 22.31 & 60.41 \\
\hline 4 & 3.9906 & 162.79 & 167.92 & 167.78 & 90.00 & 154.81 & 154.66 & 61.242 & 7.773 & 19.246 & 125.36 & 139.28 \\
\hline 5 & 2.2811 & 165.85 & 169.05 & 167.86 & 167.37 & 0 & 160.91 & 130.04 & 14.91 & 4.8034 & 153.95 & 153.92 \\
\hline 6 & 2.4221 & 164.77 & 168.64 & 167.96 & 168.20 & 159.26 & 0 & 121.35 & 14.49 & 18.781 & 150.64 & 151.93 \\
\hline 7 & -3.0409 & 169.47 & 174.86 & 170.69 & 171.39 & 166.56 & 165.52 & 0 & 12.08 & 153.87 & 171.61 & 166.52 \\
\hline 8 & -3.3570 & 171.28 & 174.47 & 171.75 & 173.60 & 169.16 & 167.91 & 169.96 & 0 & 165.09 & 173.20 & 170.44 \\
\hline 9 & -1.5303 & 170.98 & 172.60 & 171.09 & 173.49 & 171.32 & 168.19 & 165.21 & 148.4 & -90.00 & 169.50 & 168.38 \\
\hline 10 & -1.9984 & 167.67 & 174.21 & 169.35 & 168.67 & 163.53 & 162.78 & 89.66 & 7.220 & 120.80 & 90.00 & 159.64 \\
\hline 11 & -2.4009 & 171.14 & 171.74 & 168.90 & 168.69 & 164.05 & 163.08 & 141.04 & 9.816 & 110.24 & 162.13 & 0 \\
\hline
\end{tabular}

Table 6: Effect of three phase short circuit fault on line current magnitudes in p.u.

\begin{tabular}{|c|c|c|c|c|c|c|c|c|c|c|c|c|}
\hline $\begin{array}{l}\text { Line } \\
\text { no. }\end{array}$ & $\begin{array}{l}\text { Pre- } \\
\text { Fault } \\
\text { current } \\
\text { (p.u) }\end{array}$ & $\begin{array}{l}\text { When } \\
\text { fault at } \\
\text { bus no. } \\
1\end{array}$ & $\begin{array}{l}\text { When } \\
\text { fault at } \\
\text { bus no. } \\
2\end{array}$ & $\begin{array}{l}\text { When } \\
\text { fault at } \\
\text { bus no. } \\
3\end{array}$ & $\begin{array}{l}\text { When } \\
\text { fault at } \\
\text { bus no. } \\
4\end{array}$ & $\begin{array}{l}\text { When } \\
\text { fault at } \\
\text { bus no. } \\
5\end{array}$ & $\begin{array}{l}\text { When } \\
\text { fault at } \\
\text { bus no. } \\
6\end{array}$ & $\begin{array}{l}\text { When } \\
\text { fault at } \\
\text { bus no. } \\
7\end{array}$ & $\begin{array}{l}\text { When } \\
\text { fault at } \\
\text { bus no. } \\
8\end{array}$ & $\begin{array}{l}\text { When } \\
\text { fault at } \\
\text { bus no. } \\
9\end{array}$ & $\begin{array}{l}\text { When } \\
\text { fault at } \\
\text { bus } \\
\text { no. } 10\end{array}$ & $\begin{array}{l}\text { When } \\
\text { fault at } \\
\text { bus } \\
\text { no. } 11\end{array}$ \\
\hline 1 & 0.0129 & $\underline{0.2624}$ & 0.1504 & 0.1275 & 0.1178 & 0.0849 & 0.1025 & 0.1491 & 0.1440 & 0.0809 & 0.1565 & 0.2021 \\
\hline 2 & 0.0099 & $\overline{0.4512}$ & 0.1985 & 0.2203 & 0.2315 & 0.2663 & 0.2468 & 0.2014 & 0.2071 & 0.2721 & 0.1940 & 0.1523 \\
\hline 3 & 0.0219 & $\overline{0.0096}$ & 0.0740 & $\underline{0.1363}$ & 0.0495 & 0.0214 & 0.0381 & 0.0331 & 0.0101 & 0.0136 & 0.0381 & 0.0160 \\
\hline 4 & 0.0198 & 0.2615 & $\underline{0.3668}$ & 0.3036 & 0.2724 & 0.2635 & 0.2685 & 0.2048 & 0.2503 & 0.2620 & 0.2797 & 0.2622 \\
\hline 5 & 0.0152 & 0.3212 & $\overline{0.5144}$ & 0.4160 & 0.3667 & 0.3487 & 0.3589 & 0.3605 & 0.3360 & 0.3450 & 0.2794 & 0.3164 \\
\hline 6 & 0.0153 & 0.1194 & $\overline{0.1812}$ & $\underline{0.3534}$ & 0.0695 & 0.0922 & 0.0782 & 0.1437 & 0.1225 & 0.1007 & 0.1480 & 0.1267 \\
\hline 7 & 0.0052 & 0.0441 & 0.0676 & $\overline{0.0909}$ & 0.0979 & 0.0448 & $\underline{0.1731}$ & 0.0615 & 0.0589 & 0.0201 & 0.0613 & 0.0523 \\
\hline 8 & 0.0252 & 0.2099 & 0.2114 & 0.3182 & $\underline{0.3633}$ & 0.2794 & 0.3285 & 0.1918 & 0.1786 & 0.2574 & 0.1961 & 0.1974 \\
\hline 9 & 0.0198 & 0.0975 & 0.1340 & 0.1706 & $\overline{0.1841}$ & 0.0817 & 0.1388 & 0.1270 & 0.1241 & 0.0627 & 0.1261 & 0.1119 \\
\hline 10 & 0.0008 & 0.0120 & 0.0134 & 0.0366 & $\overline{0.0454}$ & 0.0946 & 0.1468 & 0.0095 & 0.0080 & 0.0354 & 0.0090 & 0.0043 \\
\hline 11 & 0.0139 & 0.2457 & 0.2681 & 0.2901 & 0.2999 & $\underline{0.5196}$ & $\overline{0.3971}$ & 0.2657 & 0.2648 & 0.2251 & 0.2648 & 0.2559 \\
\hline 12 & 0.0039 & 0.1146 & 0.2171 & 0.1427 & 0.1089 & $\overline{0.1014}$ & 0.1050 & $\underline{0.2955}$ & 0.0844 & 0.1021 & 0.1854 & 0.1206 \\
\hline 13 & 0.0047 & 0.0947 & 0.0904 & 0.0812 & 0.0771 & 0.0787 & 0.0777 & $\overline{0.0283}$ & 0.0716 & 0.0793 & $\underline{0.1459}$ & 0.0988 \\
\hline 14 & 0.0055 & 0.0307 & 0.0241 & 0.0153 & 0.0314 & 0.1122 & 0.0674 & 0.0307 & 0.0376 & $\underline{0.1244}$ & $\overline{0.0257}$ & 0.0078 \\
\hline 15 & 0.0083 & 0.1704 & 0.2341 & 0.1758 & 0.1493 & 0.1457 & 0.1472 & 0.1931 & 0.1272 & $\overline{0.1467}$ & $\underline{0.2687}$ & 0.1785 \\
\hline 16 & 0.0076 & 0.2455 & 0.1283 & 0.1189 & 0.1162 & 0.1335 & 0.1233 & 0.1166 & 0.0966 & 0.1382 & $\overline{0.1393}$ & $\underline{0.2726}$ \\
\hline 17 & 0.0012 & 0.0819 & 0.0578 & 0.0254 & 0.0088 & 0.0089 & 0.0019 & 0.0393 & 0.0098 & 0.0119 & 0.0722 & $\underline{0.0977}$ \\
\hline
\end{tabular}




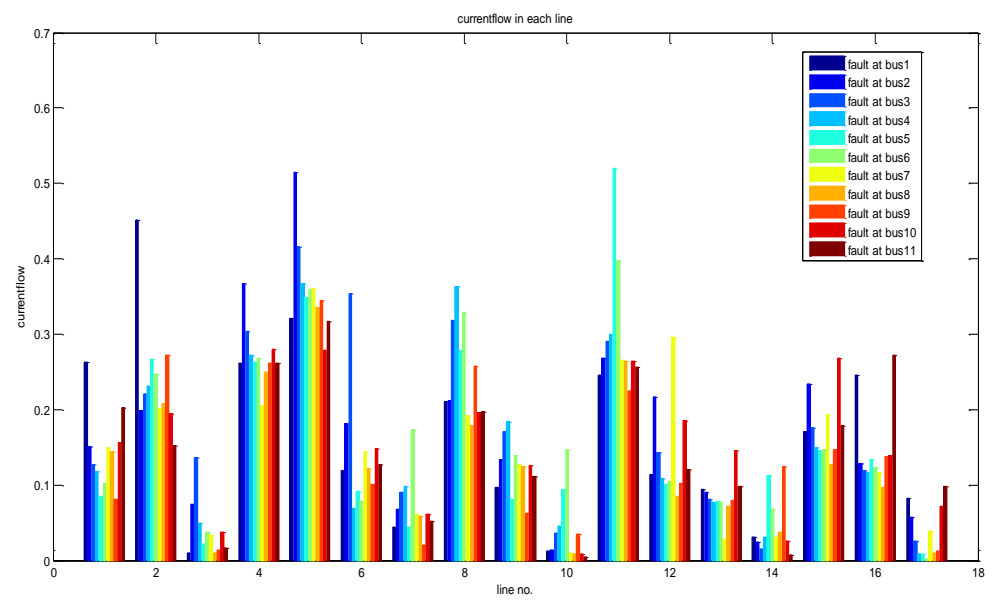

Figure 7: Graphical Representation of line currents when fault occur at different buses.

From the Table 6, it is analyzed that in line no. 1 maximum fault current flowing upto 0.2624 p.u. when there is a fault at bus no. 1 \& line no. 2 is most effected when there is a fault at bus no.1.\& line no. 3 is most effected when there is a fault at bus no.3.\& similarly check other lines and this effect is clearly shown from graphical representation of current flow in each line when short circuit fault occurs at different buses as shown in Fig.7 Now choose the SCMVA rating of Circuit Breaker for each line according to the Table 6.

Table7: SCMVA and Circuit Breaker Ratings in each line of the Standard 11 bus system.

\begin{tabular}{llc}
\hline $\begin{array}{l}\text { Line } \\
\text { no. }\end{array}$ & SCMVA & $\begin{array}{l}\text { Circuit } \\
\text { rating }\end{array}$ \\
\hline 1 & 28.07 & $\begin{array}{c}\text { Breaker } \\
\text { MVA) }\end{array}$ \\
2 & 48.28 & 30 \\
3 & 14.88 & 20 \\
4 & 40.07 & 50 \\
5 & 56.19 & 60 \\
6 & 38.70 & 40 \\
7 & 18.37 & 20 \\
8 & 38.58 & 40 \\
9 & 19.55 & 20 \\
10 & 15.35 & 20 \\
11 & 54.34 & 60 \\
12 & 29.89 & 30 \\
13 & 14.76 & 20 \\
14 & 12.31 & 20 \\
15 & 26.57 & 30 \\
16 & 26.95 & 30 \\
17 & 10.08 & 20 \\
\hline
\end{tabular}

\subsection{RESULTS FOR IEEE 30 BUS 41 TRANSMISSION LINE SYSTEM}

The load flow analysis was carried out using the Newton-Raphson load flow method then after the prefault calculations, a three phase short circuit fault was simulated on bus no.1,2,3,4,13,15,16,28,30 of the IEEE 30 bus system then calculated the fault voltage magnitude at each bus, fault voltage angle at each bus, fault currents flows in the lines, total fault current at each bus, SCMVA ratings based on the fault currents on each bus and line then the corresponding Circuit Breakers ratings are choosed. Compare the pre-fault results with post-fault results and all these results are shown below.

Table 8: Effect on voltage magnitude in p.u after short circuit occurs at buses 1,2,3,4,13,15,16,28,30.

\begin{tabular}{lllllllllll}
\hline $\begin{array}{l}\text { Bus } \\
\text { no }\end{array}$ & $\begin{array}{l}\text { Pre-fault } \\
\text { voltage } \\
\text { (p.u) }\end{array}$ & $\begin{array}{l}\text { When } \\
\text { Fault at } \\
\text { bus 1 }\end{array}$ & $\begin{array}{l}\text { When } \\
\text { Fault at } \\
\text { bus 2 }\end{array}$ & $\begin{array}{l}\text { When } \\
\text { Fault at } \\
\text { bus 3 }\end{array}$ & $\begin{array}{l}\text { When } \\
\text { Fault at } \\
\text { bus 4 }\end{array}$ & $\begin{array}{l}\text { When } \\
\text { Fault at } \\
\text { bus 13 }\end{array}$ & $\begin{array}{l}\text { When } \\
\text { Fault at } \\
\text { bus 15 }\end{array}$ & $\begin{array}{l}\text { When } \\
\text { Fault at } \\
\text { bus 16 }\end{array}$ & $\begin{array}{l}\text { When } \\
\text { Fault at } \\
\text { bus 28 }\end{array}$ & $\begin{array}{l}\text { When } \\
\text { Fault at } \\
\text { bus 30 }\end{array}$ \\
\hline 1 & $\mathbf{1 . 0 6 0 0}$ & $\mathbf{0}$ & 0.0069 & 0.0228 & 0.0333 & 0.1244 & 0.0270 & 0.0520 & 0.0312 \\
2 & $\mathbf{1 . 0 4 5 0}$ & 0.0243 & $\mathbf{0}$ & 0.0101 & 0.0221 & 0.1342 & 0.0321 & 0.0601 & 0.0213 & 0.2127 \\
3 & $\mathbf{1 . 0 2 1 3}$ & 0.0560 & 0.0388 & $\mathbf{0}$ & 0.0042 & 0.1537 & 0.0485 & 0.0789 & 0.0094 & 0.2301 \\
4 & $\mathbf{1 . 0 1 1 8}$ & 0.0647 & 0.0452 & 0.0159 & $\mathbf{0}$ & 0.1566 & 0.0510 & 0.0816 & 0.0104 & 0.2313 \\
5 & $\mathbf{1 . 0 1 0 0}$ & 0.0648 & 0.0422 & 0.0261 & 0.0123 & 0.1661 & 0.0592 & 0.0895 & 0.0137 & 0.2359 \\
6 & $\mathbf{1 . 0 0 2 7}$ & 0.0740 & 0.0533 & 0.0289 & 0.0138 & 0.1655 & 0.0583 & 0.0885 & 0.0110 & 0.2316 \\
7 & $\mathbf{0 . 9 9 7 0}$ & 0.0794 & 0.0579 & 0.0369 & 0.0222 & 0.1750 & 0.0674 & 0.0978 & 0.0206 & 0.2412 \\
8 & $\mathbf{0 . 9 9 8 9}$ & 0.0784 & 0.0577 & 0.0333 & 0.0182 & 0.1697 & 0.0622 & 0.0925 & 0.0122 & 0.2330 \\
\hline
\end{tabular}


Short Circuit Fault Analysis of Electrical Power System using MATLAB

\begin{tabular}{lllllllllll}
\hline 9 & $\mathbf{1 . 0 0 1 2}$ & 0.0755 & 0.0550 & 0.0298 & 0.0146 & 0.1499 & 0.0426 & 0.0678 & 0.0126 & 0.2260 \\
10 & $\mathbf{0 . 9 7 7 8}$ & 0.0989 & 0.0785 & 0.0528 & 0.0375 & 0.1642 & 0.0549 & 0.0784 & 0.0354 & 0.2416 \\
11 & $\mathbf{1 . 0 4 6 5}$ & 0.0304 & 0.0103 & 0.0159 & 0.0308 & 0.1048 & 0.0211 & 0.0290 & 0.0341 & 0.1908 \\
12 & $\mathbf{1 . 0 1 1 3}$ & 0.0653 & 0.0454 & 0.0180 & 0.0025 & 0.0929 & 0.0144 & 0.0387 & 0.0078 & 0.2204 \\
13 & $\mathbf{1 . 0 4 6 8}$ & 0.0300 & 0.0104 & 0.0177 & 0.0330 & $\mathbf{0}$ & 0.0394 & 0.0204 & 0.0319 & 0.1934 \\
14 & $\mathbf{0 . 9 9 2 7}$ & 0.0839 & 0.0639 & 0.0367 & 0.0212 & 0.1150 & 0.0129 & 0.0565 & 0.0234 & 0.2334 \\
15 & $\mathbf{0 . 9 8 5 3}$ & 0.0913 & 0.0713 & 0.0443 & 0.0289 & 0.1293 & $\mathbf{0}$ & 0.0656 & 0.0297 & 0.2357 \\
16 & $\mathbf{1 . 0 0 4 2}$ & 0.0724 & 0.0523 & 0.0257 & 0.0102 & 0.1158 & 0.0218 & $\mathbf{0}$ & 0.0118 & 0.2235 \\
17 & $\mathbf{0 . 9 6 9 8}$ & 0.1068 & 0.0866 & 0.0606 & 0.0452 & 0.1655 & 0.0590 & 0.0702 & 0.0439 & 0.2495 \\
18 & $\mathbf{0 . 9 6 9 7}$ & 0.1069 & 0.0868 & 0.0603 & 0.0449 & 0.1544 & 0.0317 & 0.0825 & 0.0445 & 0.2487 \\
19 & $\mathbf{0 . 9 6 3 8}$ & 0.1128 & 0.0926 & 0.0664 & 0.0510 & 0.1660 & 0.0472 & 0.0894 & 0.0501 & 0.2536 \\
20 & $\mathbf{0 . 9 6 6 5}$ & 0.1101 & 0.0899 & 0.0638 & 0.0485 & 0.1663 & 0.0498 & 0.0875 & 0.0472 & 0.2513 \\
21 & $\mathbf{0 . 9 6 4 8}$ & 0.1119 & 0.0915 & 0.0658 & 0.0505 & 0.1764 & 0.0655 & 0.0917 & 0.0480 & 0.2484 \\
22 & $\mathbf{0 . 9 6 5 5}$ & 0.1112 & 0.0908 & 0.0651 & 0.0498 & 0.1755 & 0.0642 & 0.0912 & 0.0471 & 0.2465 \\
23 & $\mathbf{0 . 9 6 8 3}$ & 0.1084 & 0.0882 & 0.0617 & 0.0464 & 0.1564 & 0.0328 & 0.0860 & 0.0449 & 0.2395 \\
24 & $\mathbf{0 . 9 5 4 6}$ & 0.1221 & 0.1018 & 0.0760 & 0.0607 & 0.1835 & 0.0678 & 0.1048 & 0.0565 & 0.2372 \\
25 & $\mathbf{0 . 9 5 8 7}$ & 0.1183 & 0.0979 & 0.0725 & 0.0573 & 0.1910 & 0.0781 & 0.1129 & 0.0472 & 0.1834 \\
26 & $\mathbf{0 . 9 3 9 9}$ & 0.1371 & 0.1167 & 0.0913 & 0.0761 & 0.2098 & 0.0964 & 0.1313 & 0.0660 & 0.1990 \\
27 & $\mathbf{0 . 9 7 0 3}$ & 0.1069 & 0.0864 & 0.0613 & 0.0461 & 0.1867 & 0.0758 & 0.1091 & 0.0324 & 0.1442 \\
28 & $\mathbf{0 . 9 9 7 1}$ & 0.0804 & 0.0597 & 0.0352 & 0.0201 & 0.1705 & 0.0626 & 0.0932 & $\mathbf{0}$ & 0.2226 \\
29 & $\mathbf{0 . 9 4 9 3}$ & 0.1278 & 0.1073 & 0.0823 & 0.0671 & 0.2077 & 0.0961 & 0.1296 & 0.0534 & 0.0791 \\
30 & $\mathbf{0 . 9 3 7 1}$ & 0.1400 & 0.1195 & 0.0945 & 0.0793 & 0.2198 & 0.1081 & 0.1415 & 0.0656 & $\mathbf{0}$ \\
\hline
\end{tabular}

Table 9: Effect on voltage angle $(\delta)$ in degree after short circuit occurs at buses 1,2,3,4,13,15,16,28,30.

\begin{tabular}{|c|c|c|c|c|c|c|c|c|c|c|}
\hline $\begin{array}{l}\text { Bus } \\
\text { no }\end{array}$ & $\begin{array}{l}\text { Pre-fault } \\
\text { angle } \\
\text { (p.u) }\end{array}$ & $\begin{array}{l}\text { When } \\
\text { Fault at } \\
\text { bus } 1\end{array}$ & $\begin{array}{l}\text { When } \\
\text { Fault at } \\
\text { bus } 2\end{array}$ & $\begin{array}{l}\text { When } \\
\text { Fault at } \\
\text { bus } 3\end{array}$ & $\begin{array}{l}\text { When } \\
\text { Fault at } \\
\text { bus } 4\end{array}$ & $\begin{array}{l}\text { When } \\
\text { Fault at } \\
\text { bus } 13\end{array}$ & $\begin{array}{l}\text { When } \\
\text { Fault at } \\
\text { bus } 15\end{array}$ & $\begin{array}{l}\text { When } \\
\text { Fault at } \\
\text { bus } 16\end{array}$ & $\begin{array}{l}\text { When } \\
\text { Fault at } \\
\text { bus } 28\end{array}$ & $\begin{array}{l}\text { When } \\
\text { Fault at } \\
\text { bus } 30\end{array}$ \\
\hline 1 & 0 & 0 & 26.2924 & 12.1461 & 8.2398 & 173.8579 & 117.9386 & 147.2798 & 19.5882 & 138.3340 \\
\hline 2 & 269 & 2652 & 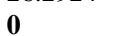 & 25.9681 & 9.2193 & 174.9636 & 135.8308 & 153.8328 & 24.4117 & 140.5312 \\
\hline 3 & -5.3230 & & 2.9516 & 0 & & 176. & 15 & & & 0999 \\
\hline 4 & -6.5336 & 30 & 175.2229 & 172.6097 & 0 & 108 & 571 & 4217 & & 145.1933 \\
\hline 5 & -6.2187 & 176.2063 & 177.3193 & 170.0837 & .7405 & 176.0970 & 158.4620 & 163.2899 & 145.4304 & 145.5689 \\
\hline 6 & 7.5981 - & 176. & 176 & & & & & 9695 & & 146.2358 \\
\hline 7 & 7.5647 & & & & & & & & & \\
\hline 8 & -8.3064 & 30 & 176 & 66 & & & & & & 336 \\
\hline 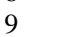 & & 9 & 176. & & & & & & & \\
\hline 10 & & & & & & & & & & \\
\hline 11 & -1 & 1 & 15 & & 2 & & & & & 136.7224 \\
\hline 12 & -1 & 7 & & & & 179.6 & & & & \\
\hline 13 & & & & & & 0 & & & & \\
\hline 14 & & 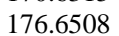 & $1^{\prime}$ & & & & & & & \\
\hline 15 & -13.4436 & 6.9104 & 177.0378 & 6.8954 & 179.1007 & 280 & . & 161.5119 & 94 & 145.9758 \\
\hline 16 & & & & & & & & 0 & & \\
\hline 17 & & & & & & & & & & \\
\hline 18 & & 4 & 17 & 3 & 179. & 177 & & & & 147.9657 \\
\hline 19 & & & & & & & & & & \\
\hline 20 & & & & & & & & & & \\
\hline 21 & & & & & & & & & & \\
\hline 22 & & 3 & 177.7 & & & & & & & 148.0283 \\
\hline 23 & & & & & & & & & & \\
\hline 24 & & & 17 & & & & & & & 148.5879 \\
\hline 25 & -1 & 177.5 & 177.8 & 177.4 & 178. & 176 & 7799 & 167.0836 & .3394 & 144.7342 \\
\hline 26 & & & & & & & & & & \\
\hline 27 & & & & & & & & & & \\
\hline 28 & -8.2282 & 8 & 176.4 & 174.6 & 175 & 176 & & & 0 & 146.8265 \\
\hline 29 & & 177.6534 & 178.0 & 177.6589 & 178.6 & 176.4669 & 16 & 168 & -179.805 & 140.7209 \\
\hline . & -15.0579 & 177.8578 & 178.2088 & 177.9611 & 178.8709 & 176.6629 & 168.3605 & 169.1519 & -179.841 & \\
\hline
\end{tabular}

Table 10: Effect of three phase short circuit fault on line current magnitudes in p.u.

\begin{tabular}{llllllllllll}
$\begin{array}{l}\text { Line } \\
\text { no. }\end{array}$ & $\begin{array}{l}\text { From- } \\
\text { to }\end{array}$ & $\begin{array}{l}\text { Pre-fault } \\
\text { linecurrent } \\
\text { (p.u) }\end{array}$ & $\begin{array}{l}\text { When } \\
\text { Fault } \\
\text { at bus } \\
\mathbf{1}\end{array}$ & $\begin{array}{l}\text { When } \\
\text { Fault } \\
\text { at bus } \\
\mathbf{2}\end{array}$ & $\begin{array}{l}\text { When } \\
\text { Fault } \\
\text { at bus } \\
\mathbf{3}\end{array}$ & $\begin{array}{l}\text { When } \\
\text { Fault } \\
\text { at bus } \\
\mathbf{4}\end{array}$ & $\begin{array}{l}\text { When } \\
\text { Fault } \\
\text { at bus } \\
\mathbf{1 3}\end{array}$ & $\begin{array}{l}\text { When } \\
\text { Fault } \\
\text { at bus } \\
\mathbf{1 5}\end{array}$ & $\begin{array}{l}\text { When } \\
\text { Fault } \\
\text { at bus } \\
\mathbf{1 6}\end{array}$ & $\begin{array}{l}\text { When } \\
\text { Fault } \\
\text { at bus }\end{array}$ & $\begin{array}{l}\text { When } \\
\text { Fault } \\
\text { at bus }\end{array}$ \\
\hline 1 & $1-2$ & $\mathbf{0 . 0 0 3 6}$ & $\mathbf{0 . 4 0 0 6}$ & 0.1136 & 0.2180 & 0.1842 & 0.1665 & 0.1726 & 0.1699 & 0.1666 & 0.1608 \\
2 & $1-3$ & $\mathbf{0 . 0 1 7 8}$ & $\mathbf{0 . 2 9 3 6}$ & 0.2346 & 0.1196 & 0.1546 & 0.1564 & 0.1626 & 0.1624 & 0.1732 & 0.1668 \\
3 & $2-4$ & $\mathbf{0 . 0 1 2 7}$ & 0.2210 & $\mathbf{0 . 2 4 7 3}$ & 0.1363 & 0.1210 & 0.1262 & 0.1340 & 0.1341 & 0.1498 & 0.1420 \\
4 & $3-4$ & $\mathbf{0 . 0 0 0 9}$ & 0.2175 & 0.1650 & $\mathbf{0 . 3 9 5 1}$ & 0.1040 & 0.0975 & 0.1087 & 0.1068 & 0.1175 & 0.1135 \\
5 & $2-5$ & $\mathbf{0 . 0 1 3 4}$ & 0.2146 & $\mathbf{0 . 2 2 3 5}$ & 0.1840 & 0.1781 & 0.1696 & 0.1696 & 0.1684 & 0.1625 & 0.1613 \\
6 & $2-6$ & $\mathbf{0 . 0 1 6 8}$ & 0.2680 & $\mathbf{0 . 2 8 7 3}$ & 0.2041 & 0.1921 & 0.1712 & 0.1736 & 0.1707 & 0.1600 & 0.1569 \\
7 & $4-6$ & $\mathbf{0 . 0 0 0 9}$ & 0.2169 & 0.1894 & 0.3040 & $\mathbf{0 . 3 2 0 8}$ & 0.2076 & 0.1809 & 0.1670 & 0.0691 & 0.0981 \\
8 & $5-7$ & $\mathbf{0 . 0 0 3 4}$ & 0.1169 & 0.1257 & 0.0882 & 0.0830 & 0.0730 & 0.0747 & 0.0734 & 0.0690 & 0.0681 \\
9 & $6-7$ & $\mathbf{0 . 0 0 0 5}$ & 0.0628 & 0.0539 & 0.0921 & 0.0977 & 0.1100 & 0.1073 & 0.1093 & 0.1136 & 0.1194 \\
\hline
\end{tabular}




\begin{tabular}{|c|c|c|c|c|c|c|c|c|c|c|c|}
\hline 10 & $6-8$ & 0.0006 & 0.1013 & 0.1011 & 0.1005 & 0.1003 & 0.0967 & 0.0937 & 0.0953 & 0.0381 & 0.0557 \\
\hline 11 & $6-9$ & 0.0126 & 0.0073 & 0.0082 & 0.0042 & 0.0037 & 0.0764 & 0.0834 & 0.1028 & 0.0079 & 0.0413 \\
\hline 12 & $6-10$ & 0.0531 & 0.0448 & 0.0454 & 0.0431 & 0.0427 & 0.0060 & 0.0072 & 0.0183 & 0.0451 & 0.0187 \\
\hline 13 & $9-11$ & 0.0094 & 0.2178 & 0.2178 & 0.2178 & 0.2178 & 0.2178 & 0.2178 & 0.2178 & 0.2178 & 0.2178 \\
\hline 14 & $9-10$ & 0.0044 & 0.2128 & 0.2138 & 0.2097 & 0.2091 & 0.1300 & 0.1222 & 0.1028 & 0.2133 & 0.1658 \\
\hline 15 & $4-12$ & 0.0269 & 0.0026 & 0.0007 & 0.0085 & 0.0096 & 0.2498 & 0.1906 & 0.1772 & 0.0142 & 0.0586 \\
\hline 16 & $12-13$ & 0.0050 & 0.2536 & 0.2536 & 0.2536 & 0.2536 & 0.6634 & 0.2536 & 0.2536 & 0.2536 & 0.2536 \\
\hline 17 & $12-14$ & 0.0071 & 0.0656 & 0.0654 & 0.0660 & 0.0660 & 0.0780 & 0.0402 & 0.0694 & 0.0642 & 0.0588 \\
\hline 18 & $12-15$ & 0.0045 & 0.1781 & 0.1774 & 0.1804 & 0.1809 & 0.2494 & 0.0981 & 0.1999 & 0.1703 & 0.1397 \\
\hline 19 & $12-16$ & 0.0020 & 0.0324 & 0.0317 & 0.0348 & 0.0353 & 0.1062 & 0.0694 & 0.1759 & 0.0274 & 0.0211 \\
\hline 20 & $14-15$ & 0.0022 & 0.0136 & 0.0135 & 0.0140 & 0.0141 & 0.0263 & 0.0236 & 0.0174 & 0.0122 & 0.0071 \\
\hline 21 & $16-17$ & 0.3540 & 0.1640 & 0.1632 & 0.1664 & 0.1668 & 0.2368 & 0.2006 & 0.3342 & 0.1586 & 0.1521 \\
\hline 22 & $15-18$ & 0.0045 & 0.0642 & 0.0638 & 0.0655 & 0.0657 & 0.1038 & 0.1303 & 0.0723 & 0.0619 & 0.0635 \\
\hline 23 & $18-19$ & 0.0009 & 0.0410 & 0.0406 & 0.0423 & 0.0425 & 0.0808 & 0.1078 & 0.0491 & 0.0387 & 0.0403 \\
\hline 24 & $19-20$ & 0.0004 & 0.0354 & 0.0358 & 0.0342 & 0.0339 & 0.0130 & 0.0404 & 0.0274 & 0.0378 & 0.0361 \\
\hline 25 & $10-20$ & 0.0047 & 0.0493 & 0.0497 & 0.0480 & 0.0477 & 0.0148 & 0.0308 & 0.0412 & 0.0517 & 0.0500 \\
\hline 26 & $10-17$ & 0.0009 & 0.0882 & 0.0890 & 0.0858 & 0.0853 & 0.0239 & 0.0523 & 0.1021 & 0.0937 & 0.1003 \\
\hline 27 & $10-21$ & 0.0013 & 0.1576 & 0.1577 & 0.1574 & 0.1573 & 0.1490 & 0.1389 & 0.1657 & 0.1531 & 0.1061 \\
\hline 28 & $10-22$ & 0.0024 & 0.0740 & 0.0740 & 0.0738 & 0.0738 & 0.0684 & 0.0619 & 0.0792 & 0.0710 & 0.0407 \\
\hline 29 & $21-22$ & 0.0000 & 0.0264 & 0.0263 & 0.0267 & 0.0267 & 0.0353 & 0.0465 & 0.0184 & 0.0310 & 0.0797 \\
\hline 30 & $15-23$ & 0.0040 & 0.0757 & 0.0752 & 0.0772 & 0.0775 & 0.1204 & 0.1455 & 0.0933 & 0.0689 & 0.0344 \\
\hline 31 & $22-24$ & 0.0026 & 0.0516 & 0.0517 & 0.0512 & 0.0511 & 0.0393 & 0.0305 & 0.0647 & 0.0446 & 0.0451 \\
\hline 32 & $23-24$ & 0.0041 & 0.0459 & 0.0454 & 0.0474 & 0.0476 & 0.0907 & 0.1164 & 0.0635 & 0.0391 & 0.0157 \\
\hline 33 & $24-25$ & 0.0032 & 0.0102 & 0.0105 & 0.0092 & 0.0090 & 0.0203 & 0.0280 & 0.0215 & 0.0252 & 0.1465 \\
\hline 34 & $25-26$ & 0.0093 & 0.0411 & 0.0411 & 0.0411 & 0.0411 & 0.0411 & 0.0411 & 0.0411 & 0.0411 & 0.0411 \\
\hline 35 & $25-27$ & 0.0036 & 0.0486 & 0.0489 & 0.0476 & 0.0474 & 0.0193 & 0.0171 & 0.0188 & 0.0632 & 0.1838 \\
\hline 36 & $28-27$ & 0.0322 & 0.0719 & 0.0723 & 0.0708 & 0.0706 & 0.0446 & 0.0360 & 0.0431 & 0.0877 & 0.2246 \\
\hline 37 & $27-29$ & 0.0146 & 0.0447 & 0.0447 & 0.0447 & 0.0447 & 0.0447 & 0.0447 & 0.0447 & 0.0447 & 0.1387 \\
\hline 38 & $27-30$ & 0.0351 & 0.0486 & 0.0486 & 0.0486 & 0.0486 & 0.0486 & 0.0486 & 0.0486 & 0.0486 & 0.2112 \\
\hline 39 & $29-30$ & 0.0104 & 0.0238 & 0.0238 & 0.0238 & 0.0238 & 0.0238 & 0.0238 & 0.0238 & 0.0238 & 0.1543 \\
\hline 40 & $8-28$ & 0.0005 & 0.0094 & 0.0095 & 0.0092 & 0.0091 & 0.0036 & 0.0021 & 0.0033 & 0.0582 & 0.0497 \\
\hline 41 & $6-28$ & 0.0008 & 0.1029 & 0.1029 & 0.1015 & 0.1011 & 0.0800 & 0.0728 & 0.0780 & 0.1769 & 0.1505 \\
\hline
\end{tabular}

From table 10, Comparing the line flow currents during fault with the pre-fault line flow currents; it is analyze that line currents increases from their normal value when there is a fault at different buses. Above table also shows that how much fault current flows in each line when there is a fault at different buses. From Table 10, in line no. 1 maximum current flowing when there is a fault occur at bus no. 1 i.e 0.4006 p.u \& in line no. 2 maximum current flowing when there is a fault at bus no.1.\& in line no. 3 maximum current flowing during fault at bus no. 2 \& in line no. 4 maximum current flowing when there is a fault at bus no. 3 \& similarly check other lines for fault at each bus of the system and choose the SCMVA rating of CB for each line acc. to above table. This effect is cleared by a graph that showing the current flow in line no. 1 when fault occur at different buses, as shown in fig.8.

From Table $11 \& 12$, it is to be noted that in the lines of IEEE 30bus 330KV system, circuit breaker ratings ranges between $10 \mathrm{MVA}$ to $70 \mathrm{MVA}$. The operating personnel can use the relay settings according to the fault current magnitude \& circuit breaker ratings.

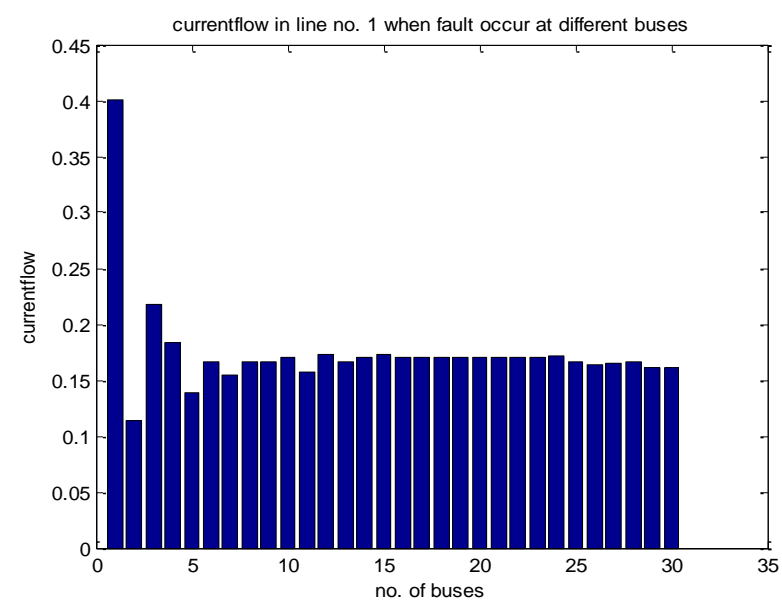

Figure 8: Graphical Representation for Current flow in line no.1 when there is a fault occur at each bus of Ieee 30 bus system. 
Table11\&12 SCMVA and Circuit Breaker Ratings in each line of the Ieee 30 bus system.

\begin{tabular}{|c|c|c|c|c|c|}
\hline Line no. & SCMVA & CB Ratings & Line no. & SCMVA & CB Ratings \\
\hline 1 & 42.46 & 45 & 22 & 15.41 & 20 \\
\hline 2 & 31.11 & 35 & 23 & 19.74 & 20 \\
\hline 3 & 25.84 & 30 & 24 & 18.50 & 20 \\
\hline 4 & 40.34 & 45 & 25 & 20.36 & 25 \\
\hline 5 & 23.35 & 25 & 26 & 18.61 & 20 \\
\hline 6 & 30.02 & 35 & 27 & 18.23 & 20 \\
\hline 7 & 32.45 & 35 & 28 & 9.076 & 15 \\
\hline 8 & 26.83 & 30 & 29 & 18.75 & 20 \\
\hline 9 & 18.33 & 20 & 30 & 15.13 & 20 \\
\hline 10 & 21.08 & 25 & 31 & 14.02 & 20 \\
\hline 11 & 21.68 & 25 & 32 & 19.62 & 20 \\
\hline 12 & 4.841 & 10 & 33 & 21.04 & 25 \\
\hline 13 & 63.58 & 65 & 34 & 38.52 & 40 \\
\hline 14 & 37.78 & 40 & 35 & 18.22 & 20 \\
\hline 15 & 25.27 & 30 & 36 & 22.38 & 30 \\
\hline 16 & 67.08 & 70 & 37 & 25.62 & 30 \\
\hline 17 & 20.89 & 25 & 38 & 20.49 & 25 \\
\hline 18 & 25.22 & 30 & 39 & 14.64 & 20 \\
\hline 19 & 17.78 & 20 & 40 & 5.919 & 10 \\
\hline 20 & 14.09 & 20 & 41 & 17.78 & 20 \\
\hline 21 & 33.55 & 35 & & & \\
\hline
\end{tabular}

Table 13\&14: Total fault current at each bus and SCMVA ratings for that fault currents on each bus.

\begin{tabular}{|c|c|c|c|c|c|}
\hline $\begin{array}{l}\text { When fault at bus } \\
\text { no. }\end{array}$ & $\begin{array}{l}\text { Fault current } \\
\text { (p.u) }\end{array}$ & SCMVA & $\begin{array}{l}\text { When fault at bus } \\
\text { no. }\end{array}$ & Fault current (p.u) & SCMVA \\
\hline 1 & 0.3727 & 39.5029 & 16 & 0.3822 & 38.3842 \\
\hline 2 & 0.3673 & 38.3869 & 17 & 0.3639 & 35.2884 \\
\hline 3 & 0.3591 & 36.6706 & 18 & 0.3768 & 36.5398 \\
\hline 4 & 0.3556 & 35.9829 & 19 & 0.3739 & 36.0412 \\
\hline 5 & 0.3642 & 36.7819 & 20 & 0.3725 & 36.0029 \\
\hline 6 & 0.3533 & 35.4265 & 21 & 0.3602 & 34.7527 \\
\hline 7 & 0.3569 & 35.5856 & 22 & 0.3606 & 34.8131 \\
\hline 8 & 0.3561 & 35.5680 & 23 & 0.3751 & 36.3251 \\
\hline 9 & 0.3687 & 36.9123 & 24 & 0.3631 & 34.6653 \\
\hline 10 & 0.3602 & 35.2240 & 25 & 0.3784 & 36.2744 \\
\hline 11 & 0.4173 & 43.6731 & 26 & 0.4308 & 40.4867 \\
\hline 12 & 0.3753 & 37.9588 & 27 & 0.3793 & 36.8030 \\
\hline 13 & 0.4098 & 42.8995 & 28 & 0.3565 & 35.5490 \\
\hline 14 & 0.3918 & 38.8945 & 29 & 0.4183 & 39.7060 \\
\hline 15 & 0.3714 & 36.5971 & 30 & 0.4230 & 39.6398 \\
\hline
\end{tabular}

\section{CONCLUSION}

From above results, it is seen that during short circuit fault voltage magnitude at faulty buses reduced to zero and current flow in the lines increases. In IEEE 11 bus system, line no. 11 is most effected i.e. in line no.11 maximum fault current flows during fault at bus no.5 and in IEEE 30 bus system, line no. 16 is most effected when there is a fault occur at bus no.13. So according to the values of fault current flows in the lines, circuit breaker rating is choosen. It is concluded that in the lines of 11 bus system circuit breaker rating ranges within 20 MVA and 60MVA and in the lines of 30 bus system circuit breaker rating ranges within 10 MVA and 70 MVA. As could be observed from the results of this study, the regular calculation of the currents which flow in the power system when a three phase fault symmetrical fault occurs and the selection of appropriate circuit breaker are required for the proper operation of the power system.

\section{ACKNOWLEDGMENT}

The authors acknowledges the support of authorities and staff of Giani Zail Singh Punjab Technical University Campus, Bathinda.

\section{APPENDIX}

TEST SYSTEM DATA

Short circuit fault analysis has been done on two standard IEEE test systems of 11 bus, 17 lines and 30 bus, 41 lines system. Line data and load data for these test systems is given below; 
Table 15: Line data for standard IEEE 11 bus system

\begin{tabular}{|l|l|l|l|}
\hline Line no. & Branch(p-q) & $\begin{array}{l}\text { Line charging } \\
\mathrm{Y}_{\mathrm{pq}}\end{array}$ & $\begin{array}{l}\text { Impedance } \\
\mathrm{Z}_{\mathrm{pq}}\end{array}$ \\
\hline 1 & $1-9$ & $\mathrm{j} 0.030$ & $0.15+\mathrm{j} 0.50$ \\
2 & $1-11$ & $\mathrm{j} 0.010$ & $0.05+\mathrm{j} 0.16$ \\
3 & $2-3$ & $\mathrm{j} 0.030$ & $0.15+\mathrm{j} 0.50$ \\
4 & $2-7$ & $\mathrm{j} 0.020$ & $0.10+\mathrm{j} 0.28$ \\
5 & $2-10$ & $\mathrm{j} 0.010$ & $0.05+\mathrm{j} 0.16$ \\
6 & $3-4$ & $\mathrm{j} 0.015$ & $0.08+\mathrm{j} 0.24$ \\
7 & $4-6$ & $\mathrm{j} 0.020$ & $0.10+\mathrm{j} 0.28$ \\
8 & $4-8$ & $\mathrm{j} 0.020$ & $0.10+\mathrm{j} 0.28$ \\
9 & $4-9$ & $\mathrm{j} 0.030$ & $0.15+\mathrm{j} 0.50$ \\
10 & $5-6$ & $\mathrm{j} 0.025$ & $0.12+\mathrm{j} 0.36$ \\
11 & $5-9$ & $\mathrm{j} 0.010$ & $0.05+\mathrm{j} 0.16$ \\
12 & $7-8$ & $\mathrm{j} 0.015$ & $0.05+\mathrm{j} 0.16$ \\
13 & $7-10$ & $\mathrm{j} 0.025$ & $0.08+\mathrm{j} 0.24$ \\
14 & $8-9$ & $\mathrm{j} 0.015$ & $0.12+\mathrm{j} 0.36$ \\
15 & $8-10$ & $\mathrm{j} 0.020$ & $0.08+\mathrm{j} 0.24$ \\
16 & $8-11$ & $\mathrm{j} 0.025$ & $0.10+\mathrm{j} 0.28$ \\
17 & $10-11$ & $0.12+\mathrm{j} 0.36$ \\
\hline
\end{tabular}

Table 16: Load data for standard IEEE 11 bus system.

\begin{tabular}{|c|c|c|c|c|c|c|}
\hline \multirow[t]{2}{*}{$\begin{array}{l}\text { Bus } \\
\text { no. }\end{array}$} & \multicolumn{2}{|c|}{$\begin{array}{l}\text { Generation } \\
\text { (p.u.) }\end{array}$} & \multicolumn{2}{|c|}{$\begin{array}{l}\text { Load } \\
\text { (p.u.) }\end{array}$} & \multicolumn{2}{|c|}{ Bus Voltage } \\
\hline & $\mathrm{P}_{\mathrm{g}}$ & $\mathrm{Q}_{\mathrm{g}}$ & $\mathrm{P}_{\mathrm{d}}$ & $\overline{Q_{d}}$ & $\mathrm{~V}$ (p.u) & $\begin{array}{c}\delta \\
(\mathrm{rad})\end{array}$ \\
\hline 1 & - & - & 0.00 & 0.00 & 1.070 & 0 \\
\hline 2 & 0.6625 & - & 0.00 & 0.00 & 1.089 & - \\
\hline 3 & 0.6625 & - & 0.00 & 0.00 & 1.095 & - \\
\hline 4 & 0.4778 & - & 0.00 & 0.00 & 1.062 & - \\
\hline 5 & 0.4778 & - & 0.00 & 0.00 & 1.046 & - \\
\hline 6 & 0.0000 & 0.0 & 0.10 & 0.02 & - & - \\
\hline 7 & 0.0000 & 0.0 & 0.40 & 0.10 & - & - \\
\hline 8 & 0.0000 & 0.0 & 0.90 & 0.45 & - & - \\
\hline 9 & 0.0000 & 0.0 & 0.70 & 0.35 & - & - \\
\hline 10 & 0.0000 & 0.0 & 0.25 & 0.05 & - & - \\
\hline 11 & 0.0000 & 0.0 & 0.25 & 0.05 & - & - \\
\hline
\end{tabular}

Table 17: Line data for standard IEEE 30 bus system

\begin{tabular}{|c|c|c|c|c|c|}
\hline \multirow{2}{*}{$\begin{array}{l}\text { Line } \\
\text { no. }\end{array}$} & \multirow{2}{*}{$\begin{array}{l}\text { From } \\
\text { Bus }\end{array}$} & \multirow{2}{*}{$\begin{array}{l}\text { To } \\
\text { Bus }\end{array}$} & \multicolumn{2}{|c|}{ Line Impedance } & \multirow{2}{*}{$\begin{array}{l}\text { Half Line } \\
\text { Charging } \\
\text { Susceptance } \\
\text { (p.u.) }\end{array}$} \\
\hline & & & R(p.u.) & X(p.u.) & \\
\hline 1 & 1 & 2 & 0.0192 & 0.0575 & 0.0264 \\
\hline 2 & 1 & 3 & 0.0452 & 0.1652 & 0.0204 \\
\hline 3 & 2 & 4 & 0.0570 & 0.1737 & 0.0184 \\
\hline 4 & 3 & 4 & 0.0132 & 0.0379 & 0.0042 \\
\hline 5 & 2 & 5 & 0.0472 & 0.1983 & 0.0209 \\
\hline 6 & 2 & 6 & 0.0581 & 0.1763 & 0.0187 \\
\hline 7 & 4 & 6 & 0.0119 & 0.0414 & 0.0045 \\
\hline 8 & 5 & 7 & 0.0460 & 0.1160 & 0.0102 \\
\hline 9 & 6 & 7 & 0.0267 & 0.0820 & 0.0085 \\
\hline 10 & 6 & 8 & 0.0120 & 0.0420 & 0.0045 \\
\hline 11 & 6 & 9 & 0 & 0.2080 & 0 \\
\hline 12 & 6 & 10 & 0 & 0.5560 & 0 \\
\hline 13 & 9 & 11 & 0 & 0.2080 & 0 \\
\hline 14 & 9 & 10 & 0 & 0.1100 & 0 \\
\hline 15 & 4 & 12 & 0 & 0.2560 & 0 \\
\hline 16 & 12 & 13 & 0 & 0.1400 & 0 \\
\hline 17 & 12 & 14 & 0.1231 & 0.2559 & 0 \\
\hline 18 & 12 & 15 & 0.0662 & 0.1304 & 0 \\
\hline 19 & 12 & 16 & 0.0945 & 0.1987 & 0 \\
\hline 20 & 14 & 15 & 0.2210 & 0.1997 & 0 \\
\hline 21 & 16 & 17 & 0.0524 & 0.1923 & 0 \\
\hline 22 & 15 & 18 & 0.1073 & 0.2185 & 0 \\
\hline 23 & 18 & 19 & 0.0639 & 0.1292 & 0 \\
\hline 24 & 19 & 20 & 0.0340 & 0.0680 & 0 \\
\hline 25 & 10 & 20 & 0.0936 & 0.2090 & 0 \\
\hline 26 & 10 & 17 & 0.0324 & 0.0845 & 0 \\
\hline
\end{tabular}




\begin{tabular}{|l|l|l|l|l|l|}
\hline 27 & 10 & 21 & 0.0348 & 0.0749 & 0 \\
28 & 10 & 22 & 0.0727 & 0.1499 & 0 \\
29 & 21 & 22 & 0.0116 & 0.0236 & 0 \\
30 & 15 & 23 & 0.1000 & 0.2020 & 0 \\
31 & 22 & 24 & 0.1150 & 0.1790 & 0 \\
32 & 23 & 24 & 0.1320 & 0.2700 & 0 \\
33 & 24 & 25 & 0.1885 & 0.3292 & 0 \\
34 & 25 & 26 & 0.2544 & 0.3800 & 0 \\
35 & 25 & 27 & 0.1093 & 0.2087 & 0 \\
36 & 28 & 27 & 0 & 0.3960 & 0 \\
37 & 27 & 29 & 0.2198 & 0.4153 & 0 \\
38 & 27 & 30 & 0.3202 & 0.6027 & 0 \\
39 & 29 & 30 & 0.2399 & 0.4533 & 0 \\
40 & 8 & 28 & 0.0636 & 0.2000 & 0.0214 \\
41 & 6 & 28 & 0.0169 & 0.0599 & 0.0065 \\
\hline
\end{tabular}

Table 18: Load data for standard IEEE 30 bus system

\begin{tabular}{|c|c|c|c|c|c|c|}
\hline \multirow[t]{2}{*}{$\begin{array}{l}\text { Bus } \\
\text { no. }\end{array}$} & \multicolumn{2}{|c|}{$\begin{array}{l}\text { Generation } \\
\text { (p.u.) }\end{array}$} & \multicolumn{2}{|l|}{$\begin{array}{l}\text { Load } \\
\text { (p.u.) }\end{array}$} & \multicolumn{2}{|c|}{ Bus Voltage } \\
\hline & $P_{g}$ & $\mathrm{Q}_{\mathrm{g}}$ & $\mathrm{P}_{\mathrm{d}}$ & $\mathrm{Qd}_{\mathrm{d}}$ & V (p.u) & $\begin{array}{c}\delta \\
(\mathrm{deg})\end{array}$ \\
\hline 1 & 1.3848 & -0.029 & 0.000 & 0.000 & 1.060 & 0.0 \\
\hline 2 & 0.400 & 0.5 & 0.217 & 0.127 & 1.045 & 0.0 \\
\hline 3 & 0.000 & 0.000 & 0.024 & 0.012 & 1.000 & 0.0 \\
\hline 4 & 0.000 & 0.000 & 0.076 & 0.016 & 1.060 & 0.0 \\
\hline 5 & 0.000 & 0.37 & 0.942 & 0.19 & 1.010 & 0.0 \\
\hline 6 & 0.000 & 0.000 & 0.000 & 0.000 & 1.000 & 0.0 \\
\hline 7 & 0.000 & 0.000 & 0.228 & 0.109 & 1.000 & 0.0 \\
\hline 8 & 0.000 & 0.373 & 0.3 & 0.3 & 1.010 & 0.0 \\
\hline 9 & 0.000 & 0.000 & 0.000 & 0.000 & 1.000 & 0.0 \\
\hline 10 & 0.000 & 0.000 & 0.058 & 0.02 & 1.000 & 0.0 \\
\hline 11 & 0.000 & 0.162 & 0.000 & 0.000 & 1.082 & 0.0 \\
\hline 12 & 0.000 & 0.000 & 0.112 & 0.075 & 1.000 & 0.0 \\
\hline 13 & 0.000 & 0.106 & 0.000 & 0.000 & 1.071 & 0.0 \\
\hline 14 & 0.000 & 0.000 & 0.062 & 0.016 & 1.000 & 0.0 \\
\hline 15 & 0.000 & 0.000 & 0.082 & 0.025 & 1.000 & 0.0 \\
\hline 16 & 0.000 & 0.000 & 0.035 & 0.018 & 1.000 & 0.0 \\
\hline 17 & 0.000 & 0.000 & 0.09 & 0.058 & 1.000 & 0.0 \\
\hline 18 & 0.000 & 0.000 & 0.032 & 0.009 & 1.000 & 0.0 \\
\hline 19 & 0.000 & 0.000 & 0.095 & 0.034 & 1.000 & 0.0 \\
\hline 20 & 0.000 & 0.000 & 0.022 & 0.007 & 1.000 & 0.0 \\
\hline 21 & 0.000 & 0.000 & 0.175 & 0.112 & 1.000 & 0.0 \\
\hline 22 & 0.000 & 0.000 & 0.000 & 0.000 & 1.000 & 0.0 \\
\hline 23 & 0.000 & 0.000 & 0.032 & 0.016 & 1.000 & 0.0 \\
\hline 24 & 0.000 & 0.000 & 0.087 & 0.067 & 1.000 & 0.0 \\
\hline 25 & 0.000 & 0.000 & 0.000 & 0.000 & 1.000 & 0.0 \\
\hline 26 & 0.000 & 0.000 & 0.035 & 0.023 & 1.000 & 0.0 \\
\hline 27 & 0.000 & 0.000 & 0.000 & 0.000 & 1.000 & 0.0 \\
\hline 28 & 0.000 & 0.000 & 0.000 & 0.000 & 1.000 & 0.0 \\
\hline 29 & 0.000 & 0.000 & 0.024 & 0.009 & 1.000 & 0.0 \\
\hline 30 & 0.000 & 0.000 & 0.106 & 0.019 & 1.000 & 0.0 \\
\hline
\end{tabular}

\section{REFERENCES}

[1] Adnan Kakilli, System analysis with the MVA method for Symmetrical Three-Phase Faults, TEM Journal, volume 2, number 1,pp-51-56,2013.

[2] A.Muhammad, Simulation of Different Types of Faults on Northern Iraq Power System, IGEC VI - 2011 - 028.

[3] Okelola, M.O, Yussuf, A.A, and Awosope, C.O.A.,Fault Analysis: An Application of Venin's Method to 330kv Transmission Grid System in Nigeria, LAUTECH Journal of Engineering and Technology, Vol.3, No.1, pp.30-43,2005.

[4] I.J. Nagrath, and D.P.Kothari, Modern Power System Analysis, Tata McGraw-Hill Publishing Company Limited, New Delhi,India,2012.

[5] V.K.Mehta and R.Mehta, Principles of Power Systems, S.Chand and Company Limited, New Delhi, India, 2010.

[6] Ahmed Waleed K.," Advantages and Disadvantages of using MATLAB/ode45 for solving Differential Equations in Engineering Applications", International Journal of Engineering, Vol.7, Issue 1, Page 25-31, 2013.

[7] Raj Kumar Bansal, Ashok Kumar Goel and Manoj Kumar Sharma, MATLAB and its Applications in Engineering, Dorling Kindersley (India) Pvt. Ltd., licenses of Pearson Education in South Asia, New Delhi, 2012.

[8] Dharamjit and D.K.Tanti, Load Flow Analysis on IEEE 30 Bus System, International Journal of Scientific and Research Publications, Volume 2, Issue 11, November 2012

[9] Pushp Raj, Load Flow and Short Circuit Analysis of 400/220kv Substation, International Journal of Creative Research Thoughts, Volume 1, Issue.4, April 2013.

[10] V.J.Rathod, Prof. G.R.Patel, Short Circuit Analysis on 400kv Sub-Station Soja, International Journal of Engineering Research and Development, Volume 6, Issue 11, April 2013, pp.62-67

[11] B.R.Gupta, Power System Analysis and Design, S.Chand and Company Ltd., New Delhi, India, 2006.

[12] Ravi Kumar Tiwari, Nidhi Singh, Ambarish Kumar, D Suresh Babu, Analysis of Symmetrical Fault with Fault Impedance in Power System, Global Research Analysis(International research paper), Volume:2, Issue:1, January 2013.

[13] B.Ravinderanath.M.Chander,Power System Protection and Switchgear, New age international publishers, New Delhi, 2012 